\title{
RNA-Seq analysis of soft rush (Juncus effusus): transcriptome sequencing, de novo assembly, annotation, and polymorphism identification
}

Muhammad Arslan ${ }^{1,2}$, Upendra Kumar Devisetty ${ }^{3}$, Martin Porsch ${ }^{4,5}$, Ivo Große $^{4,6}$, Jochen A. Müller ${ }^{1 *}$ (D) and Stefan G. Michalski ${ }^{7}$

\begin{abstract}
Background: Juncus effusus L. (family: Juncaceae; order: Poales) is a helophytic rush growing in temperate damp or wet terrestrial habitats and is of almost cosmopolitan distribution. The species has been studied intensively with respect to its interaction with co-occurring plants as well as microbes being involved in major biogeochemical cycles. J. effusus has biotechnological value as component of Constructed Wetlands where the plant has been employed in phytoremediation of contaminated water. Its genome has not been sequenced.

Results: In this study we carried out functional annotation and polymorphism analysis of de novo assembled RNASeq data from 18 genotypes using 249 million paired-end Illumina HiSeq reads and 2.8 million 454 Titanium reads. The assembly comprised 158,591 contigs with a mean contig length of $780 \mathrm{bp}$. The assembly was annotated using the dammit! annotation pipeline, which queries the databases OrthoDB, Pfam-A, Rfam, and runs BUSCO (Benchmarking Single-Copy Ortholog genes). In total, 111,567 contigs (70.3\%) were annotated with functional descriptions, assigned gene ontology terms, and conserved protein domains, which resulted in 30,932 nonredundant gene sequences. Results of BUSCO and KEGG pathway analyses were similar for $\mathrm{J}$. effusus as for the wellstudied members of the Poales, Oryza sativa and Sorghum bicolor. A total of 566,433 polymorphisms were identified in transcribed regions with an average frequency of 1 polymorphism in every 171 bases.
\end{abstract}

Conclusions: The transcriptome assembly was of high quality and genome coverage was sufficient for global analyses. This annotated knowledge resource can be utilized for future gene expression analysis, genomic feature comparisons, genotyping, primer design, and functional genomics in J. effusus.

Keywords: Juncus effusus, Soft rush, Helophyte, Wetlands, Transcriptome annotation, RNA-Seq, Polymorphism

\section{Background}

Juncus effusus L. (common, soft or mat rush) is an almost cosmopolitan monocotyledonous C3 plant that can grow abundantly in temperate wetlands, riparian strips, and other damp or wet terrestrial habitats [1]. The plant can vary substantially in morphological traits across its worldwide distributional range leading to the description of several subspecies. In Europe, only J. effusus ssp. effusus is

\footnotetext{
* Correspondence: jochen.mueller@ufz.de

${ }^{1}$ Department Environmental Biotechnology, Helmholtz Centre for Environmental Research - UFZ, Permoserstr, 15 Leipzig, Germany Full list of author information is available at the end of the article
}

known to occur but at least two genetically distinct cryptic lineages within the taxa have been found recently [2].

The plant grows in dense tufts and is able to reproduce by producing abundant seeds, which are easily dispersed, as well as via rhizomes, rendering the species an efficient colonizer [3]. The rhizomes as well as the shoots of this helophyte are characterized by forming aerenchyma for channeling air into the roots. This structural feature allows $J$. effusus to thrive in waterlogged environments [4-6]. The plant has multifarious effects on major element cycles in wetlands [7]. For example, radial oxygen loss can reduce $\mathrm{CH}_{4}$ production and increase

(c) The Author(s). 2019 Open Access This article is distributed under the terms of the Creative Commons Attribution 4.0 International License (http://creativecommons.org/licenses/by/4.0/), which permits unrestricted use, distribution, and 
$\mathrm{CH}_{4}$ oxidation in the rhizosphere [8-10]; on the other side, the input of organic carbon (root exudates and plant litter) can enhance methanogenesis [11, 12] and the aerenchyma can act as conduit for methane emission from organic-rich soils into the atmosphere [13].

Interactions of $J$. effusus with rhizospheric microbial communities as well as co-occurring plant species are exploited in ecotechnological applications such as Constructed Wetlands (CWs) [14]. CWs are means for wastewater treatment mirroring chemical transformation processes in natural wetlands to remove organic and inorganic contaminants from water [5]. Based on these characteristics J. effusus has been employed as a model plant in basic and applied research on wetland ecosystems [15-18]. The stem is of economic value as commodity for various woven products [19]. In addition, J. effusus has some medicinal properties and produces a variety of bioactive compounds [20, 21]. The pith of the stem, Junci Medulla, has been used in Chinese and other traditional medicines [22].

Understanding and quantifying genetic diversity within $J$. effusus is fundamental in predicting evolutionary pathways under changing environmental conditions. Marker systems such as single nucleotide polymorphisms (SNPs) and insertions/deletions (INDELs) have several advantages over conventional genetic markers. This includes their high genomic abundance, a co-dominant expression, and being mostly phenotypically neutral in nature [23]. Although a strong degree of genetic structuring has been suggested for J. effusus [2, 24], very little information is available at the molecular level. The species is diploid $(2 n=42)$ and has a relatively small genome with a measured DNA $1 C$-value of $0.3 \mathrm{pg}$ [25]. Based on this value the genome has an estimated size of approximately $270 \mathrm{Mbp}$, i.e. in between the genome sizes of Arabidopis thaliana (Arabidopsis Genome Initiative, 2000) and Oryza sativa [26, 27]. Plastome sequence data are available [28].

The aim of the present study was to develop a molecular database of $J$. effusus for enhanced research on natural and engineered wetland ecosystem functioning. To this end we employed RNA-Seq to record gene transcription in adult roots and shoots of 18 genotypes. The transcriptome was de novo assembled and annotated. Ortholog comparisons with phylogenetic relatives were carried out and the genetic diversity among the genotypes was evaluated based on a SNP analysis. The genomic information thus obtained will be of benefit for studies on wetland ecosystems and will foster further evolutionary studies on the Poales.

\section{Results}

\section{Assembly of the J. effusus transcriptome}

The overall process of transcriptome sequencing, assembly, annotation, ortholog clustering and validation of the assembly is summarized in Fig. 1. Illumina and 454 sequencing generated 108,600,750 clean reads comprising a total of $47 \mathrm{~Gb}$, which was considered as good transcriptome coverage of the estimated genome size of around $270 \mathrm{Mb}$. The reads were de novo assembled using Trinity [29] and Mira [30, 31]. Quality analysis of the Trinity assembly with the software TransRate computed an optimized score of 0.34 , which was better than the score for about $50 \%$ of 155 sampled de novo assembled transcriptomes [29]. CD-HIT [32, 33] was used to remove redundant sequences, which resulted in 158,591 contigs with lengths ranging between $200 \mathrm{bp}$ to $18.5 \mathrm{~kb}$. The average contig length was $780 \mathrm{bp}$, and N50 was $255 \mathrm{bp}$.

BUSCO v3 [34] was run on the J. effusus assembly as well as on previously assembled and annotated transcriptomes of $O$. sativa and S. bicolor to determine whether the genome coverage was sufficiently high to allow for comprehensive analyses. BUSCO results for the three species were very similar. Out of 429 single-copy ortholog genes common to the Eukaryota lineage there were 81, 82, and $78 \%$ complete single-copy BUSCOs, 42,26 , and $24 \%$ duplicated BUSCOs, 8.8, 4.1, and 6\% fragmented BUSCOs, and 9.5, 12, and 15\% missing BUSCOs respectively for $J$. effusus, S. bicolor and O. sativa.

\section{Constructing and annotating gene models}

The assembled transcripts were annotated using Camille Scott's dammit! annotation pipeline (https:/github.com/ camillescott/dammit). Gene model building using Transdecoder [35] predicted 120,343 likely coding regions (75.8\% of all contigs) among which 79,203 (49.4\%) contained a stop codon. There were 62,745 (39.6\%) predicted coding regions that matched to the protein family database Pfam [36, 37], whereas a LAST search found that 67,835 predicted coding regions $(42.8 \%)$ matched to the OrthoDB database $[38,39]$. In addition, 3385 predicted coding regions (2.13\%) matched to the Rfam database for non-coding RNAs [40]. In total, 111,567 contigs (70.3\%) were annotated when combining results of all searches. The annotation features included putative nucleotide and protein matches, five- and three-prime UTRs, exons, mRNA, as well as start and stop codons.

To ensure further that the assembly was of high quality, we compared genomic features both statistically and manually with previously well-annotated transcriptomes of $S$. bicolor and O. sativa. GO analysis by InterProScan allowed classification of annotated transcripts into different functional groups. A total of 42,739 sequences (38.3\% of all annotated contigs) were GO annotated out of the categories Molecular Functions, Cellular Components, and Biological Processes. The WEGO [41] plot for GO terms revealed that Molecular Functions was the dominant category (50.7\% of all GO-annotations) followed by Biological Processes (35.7\%) and Cellular Components (13.6\%). Highly represented GO terms within Molecular Functions were 


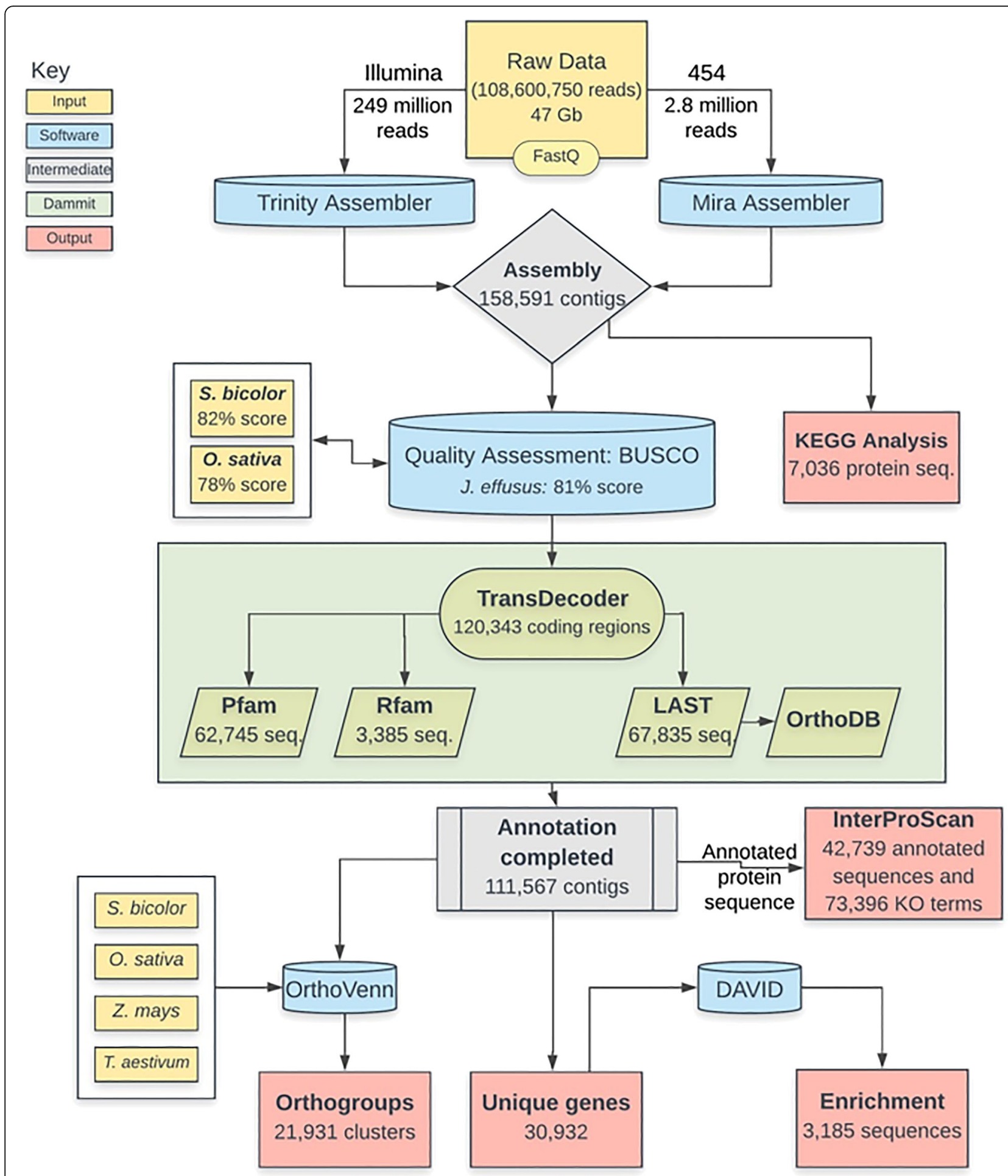

Fig. 1 The overall process of transcriptome assembly, functional annotation, GO enrichment, orthologs clustering and validation

'binding' (GO:0005488) and 'catalytic activity' (GO: 0003824); in the Biological Processes ontology group it were 'cells' (GO:0005623), 'cellular process' (GO:0009987), and 'biological regulation' (GO:0065007); and 'cellular parts' (GO:0044464) and 'organelles' (GO:0043226) in the
Cellular Components ontology. The GO terms of the assembled transcriptome were compared with those of $S$. bicolor and O. sativa (Fig. 2). The results revealed a similar functional distribution with both reference transcriptomes, suggesting similar gene complements between $J$. effusus 


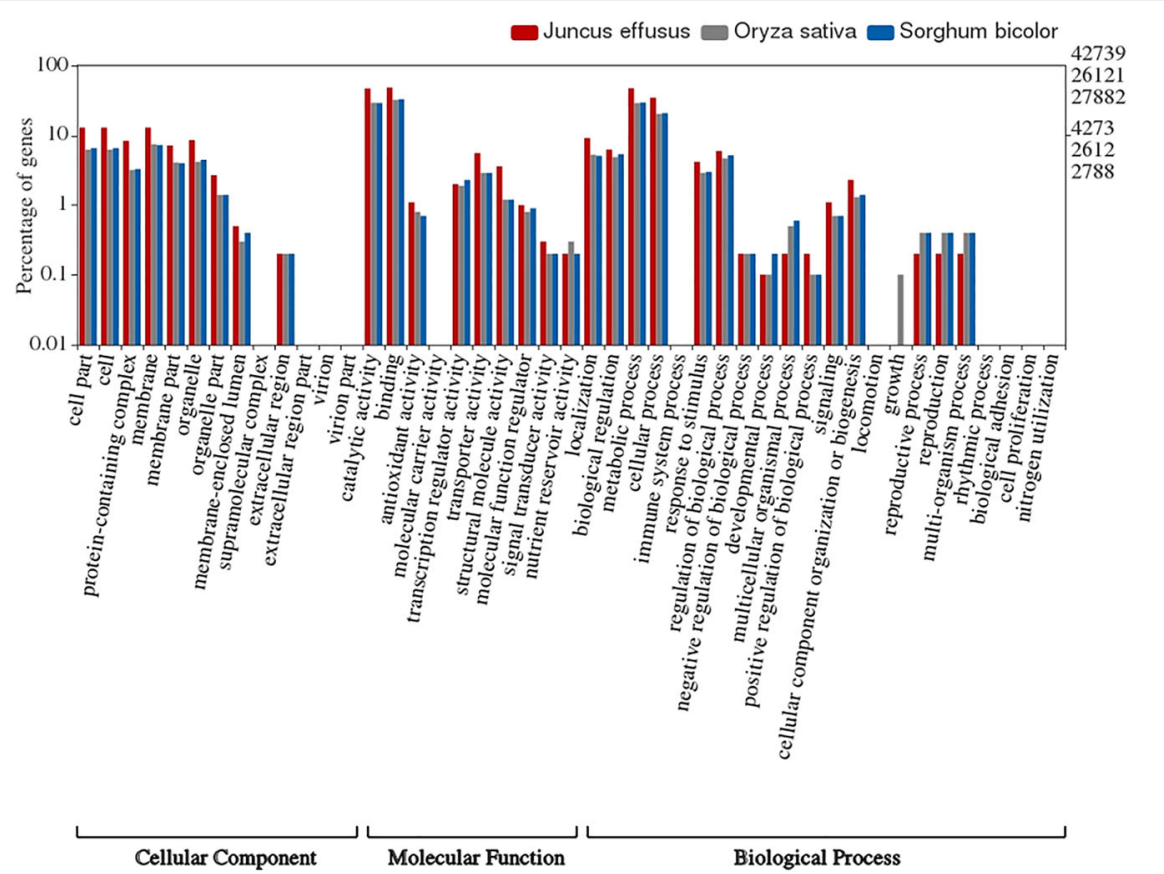

Fig. 2 Histogram of level GO term assignments for J. effusus, S. bicolor, and O. sativa annotated gene models. Results are summarized for three main GO categories, Cellular Component, Molecular Function, Biological Process

and its relatives. Minor contributions of 'antioxidant activity' (GO:0016209), 'extracellular region' (GO:0005576), 'extracellular part' (GO:0044421), and 'viral reproduction' (GO:0016032) were observed for J. effusus, while those categories were missing for S. bicolor and O. sativa.

KEGG analysis assigned enzyme commission (EC) numbers to 7036 protein sequences belonging to 380 different pathways. The KEGG category 'metabolic pathways' contained the majority of annotated proteins (851 members, $12.1 \%$ ), followed by 'biosynthesis of secondary metabolites' (395 members, 5.61\%). To evaluate further the qualitative accuracy of the functional annotation, we manually checked the completeness of the fundamental pathways photosynthesis, oxidative phosphorylation, glycolysis/gluconeogenesis, citrate cycle, pentose phosphate pathway, amino acid metabolism, and information processing. In addition, we checked the completeness of pathways involved in waterlogging [42]. All of those pathways were fully covered in the transcriptome.

Clusters of orthologous gene (COG) analysis of $J$. effusus revealed the presence of 21,931 clusters, out of which 10,296 were shared among S. bicolor, O. sativa, and Zea mays (Fig. 3). These clusters involve proteins related to carboxylation and oxygenation, glycosylation, integral membrane components, nuclear mechanisms such as chromatin binding, cytoplasm and chloroplast integrities, and several other putative uncharacterized proteins. Further analyses of GO terms revealed a significant enrichment for the proteins related to electron carrier activities in the mitochondrial matrix (e.g., GO: 0019243), photosystem II assembly (e.g., GO:0010207), transcription from plastid promoter (e.g., GO:0042793), regulation of protein dephosphorylation (e.g., GO: 0035304), and hydrogen peroxide biosynthetic process (e.g., GO:0050665). The three members of the Poaceae had more similarities to each other than to J. effusus, which matches the topology of the phylogenetic tree based on plastome sequences [28]. Overall, 9872 clusters were unique for $J$. effusus, and included proteins involved in chloroplastic mechanisms, plasma membrane functioning, disease resistance, phytohormone productions for stress-ripening, and ion binding.

As a final quality control, we conducted Gene Set Enrichment Analysis (GSEA) with DAVID [43]. Results of GSEA were consistent with the KEGG findings. A complete list of enriched sequences and number of KEGG orthology (KO) hits for J. effusus, S. bicolor, O. sativa and $Z$. mays is presented in Table 1. Sequences of $J$. effusus with redundant $\mathrm{KO}$ terms likely originating from paralogous genes and orthologues in the various genotypes were combined to a total of 30,932 gene sequences with matching hits to proteins $\left(\mathrm{E}<1 \mathrm{e}^{-6}\right)$. Among these sequences there were 3185 enriched sequences $(10.2 \%)$ of which most belonged to the subgroups of metabolic pathways (1407 sequences, 44.1\%), biosynthesis of secondary metabolites (1140 sequences, 35.8\%), biosynthesis of amino acids (156 sequences, 4.89\%), oxidative phosphorylation (114 sequences, 


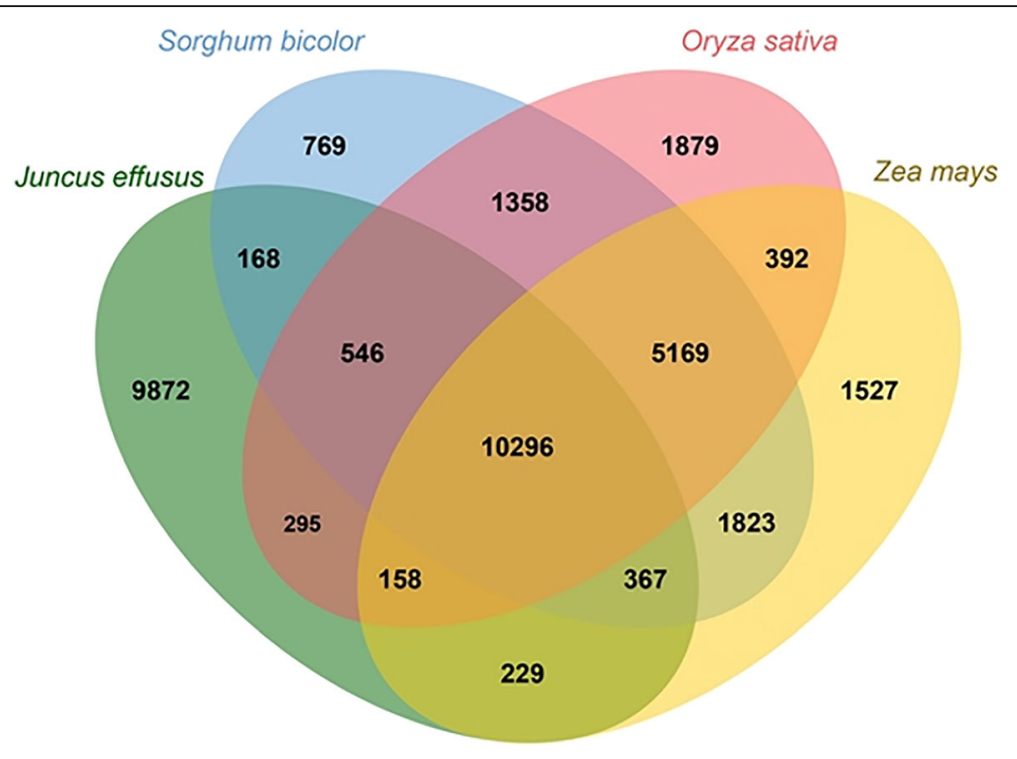

Size of each list

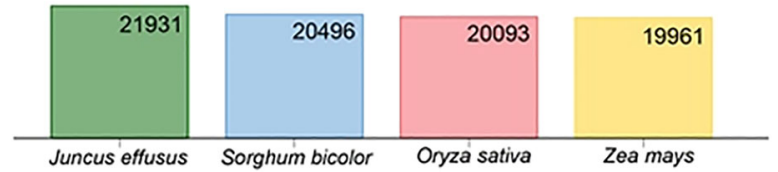

Fig. 3 Comparisons of the core orthologous gene clusters among J. effusus, O. sativa, Z. mays, and S. bicolor

3.57\%), amino sugar and nucleotide sugar metabolism (113 sequences, $3.54 \%$ ). Sequences grouping into the category genetic information processing accounted for 322 sequences $(1.04 \%)$ and included the enriched categories ribosomes (276 sequences, 85.7\%) and protein export (46 sequences, 14.2\%). By contrast, environmental information processing (EIP) contained no enriched KEGG pathways for J. effusus (although the EIP pathways were complete as mentioned above). All pathways enriched for in J. effusus were also enriched for in $S$. bicolor except porphyrin and chlorophyll metabolism, which was only enriched in J. effusus.

\section{Detection of nucleotide polymorphisms}

We identified 566,433 polymorphisms (478,627 SNPs and 87,806 INDELs) in 99,692 contigs. On average over the whole transcriptome, one polymorphism was identified in $174 \mathrm{bp}$. This relative abundance is similar to SNPs frequency in O. sativa (1 per $147 \mathrm{bp}$ ) [44] and Z. mays (1 per $200 \mathrm{bp)} \mathrm{[45].} \mathrm{Among} \mathrm{the} \mathrm{contigs} \mathrm{having} \mathrm{at} \mathrm{least}$ one polymorphism, 35,429 were assigned to KO terms and comprised 3521 unique $\mathrm{KO}$ identifiers. The polymorphism distribution among gene functions was nonrandom. Ten out of the top $20 \mathrm{KO}$ IDs with the highest polymorphism frequencies (ranging from 9 to 49 per $\mathrm{KO}$ ID) were associated with plant defense mechanisms and wound healing (disease resistance protein RPM1, peroxidase, chalcone synthase, ATP-binding cassette of multidrug resistant transporter, phenylalanine ammonia-lyase, laccase, glutathione $S$-transferase, trans-resveratrol di-Omethyltransferase, cinnamyl-alcohol dehydrogenase, ionotropic glutamate receptor). Most of the remaining KO IDs with $\geq 5$ polymorphisms (50 IDs in total) were involved in cytoskeleton formation, repair mechanisms associated with replication, transcription and translation, as well as metabolism of the small amino acids Gly, Met, Cys, Ser and Thr.

In order to experimentally confirm the suitable of the SNP dataset for genotyping of J. effusus, we selected 44 SNPs for loci amplification via PCR and subsequent Sanger sequencing. The loci chosen were predicted to be associated with nitrogen assimilation (Table 2), which is an important function in wastewater treatment via constructed wetlands. Out of those selected a total of 35 (80\%) loci could be successfully PCR-amplified and thus the respective SNPs confirmed by sequencing.

\section{Discussion}

In the past the extensive research on and the manifold biotechnological applications of the common wetland plant $J$. effusus were carried out without omics-based knowledge. Information on nucleic acid sequences was 
Table 1 Genes enriched for KEGG and number of KO hits for Juncus effusus, Sorghum bicolor, Oryza sativa, and Zea mays

\begin{tabular}{|c|c|c|c|c|c|c|c|c|c|}
\hline \multirow[b]{2}{*}{ Ranking } & \multirow[b]{2}{*}{ KEGG pathway } & \multicolumn{2}{|l|}{ J. effusus } & \multicolumn{2}{|l|}{ S. bicolor } & \multicolumn{2}{|l|}{ O. sativa } & \multicolumn{2}{|l|}{ Z. mays } \\
\hline & & KEGG hits & KO hits & KEGG hits & KO hits & KEGG hits & KO hits & KEGG hits & $\mathrm{KO}$ hits \\
\hline 1. & Metabolism & & & & & & & & \\
\hline 1.0 & Global and overview maps & & & & & & & & \\
\hline 1100 & Metabolic pathways & 1407 & $855>$ & 1431 & 865 & 1369 & 817 & 1793 & 850 \\
\hline 1110 & Biosynthesis of secondary metabolites & 833 & 396 & 844 & 395 & 776 & 397 & 1033 & 400 \\
\hline 1130 & Biosynthesis of antibiotics & 307 & 193 & 312 & 194 & 352 & 192 & 462 & 194 \\
\hline 1200 & Carbon metabolism & - & - & - & - & 226 & 90 & 263 & 90 \\
\hline 1230 & Biosynthesis of amino acids & 156 & 98 & 157 & 98 & 189 & 97 & - & - \\
\hline 1.1 & Carbohydrate metabolism & & & & & & & & \\
\hline 00010 & Glycolysis / Gluconeogenesis & 92 & 33 & 94 & 33 & 112 & 32 & 134 & 33 \\
\hline 00020 & Citrate cycle (TCA cycle) & - & - & - & - & 49 & 20 & - & - \\
\hline 00030 & Pentose phosphate pathway & - & - & - & - & 46 & 17 & - & - \\
\hline 00053 & Ascorbate and aldarate metabolism & - & - & - & - & 37 & 16 & - & - \\
\hline 00500 & Starch and sucrose metabolism & - & - & - & - & 107 & 30 & - & - \\
\hline 00520 & Amino sugar and nucleotide sugar metabolism & 113 & 40 & 114 & 40 & 105 & 40 & - & - \\
\hline 00620 & Pyruvate metabolism & - & - & - & - & 73 & 27 & - & - \\
\hline 1.2 & Energy metabolism & & & & & & & & \\
\hline 00190 & Oxidative phosphorylation & 114 & 86 & 118 & 91 & - & - & 129 & 86 \\
\hline 00195 & Photosynthesis & - & - & - & - & 75 & 35 & - & - \\
\hline 00710 & Carbon fixation in photosynthetic organisms & - & - & - & - & 70 & 25 & - & - \\
\hline 1.3 & Lipid metabolism & & & & & & & - & - \\
\hline 00073 & Cutin, suberine and wax biosynthesis & - & - & - & - & - & - & 28 & 8 \\
\hline 00100 & Steroid biosynthesis & - & - & - & - & - & - & 38 & 18 \\
\hline 00591 & Linoleic acid metabolism & - & - & - & - & - & - & 15 & 4 \\
\hline 1.5 & Amino acid metabolism & & & & & & & & \\
\hline 00260 & Glycine, serine and threonine metabolism & 52 & 34 & 52 & 34 & - & - & - & - \\
\hline 00330 & Arginine and proline metabolism & - & - & - & - & - & - & 62 & 24 \\
\hline 00350 & Tyrosine metabolism & - & - & - & - & - & - & 40 & 18 \\
\hline 1.6 & Metabolism of other amino acids & & & & & & & & \\
\hline 00480 & Glutathione metabolism & 98 & 18 & 98 & 18 & - & - & - & - \\
\hline 1.7 & Glycan biosynthesis and metabolism & & & & & & & & \\
\hline 00510 & $\mathrm{~N}$-Glycan biosynthesis & - & - & - & - & - & - & 44 & 31 \\
\hline 1.8 & Metabolism of cofactors and vitamins & & & & & & & & \\
\hline 00730 & Thiamine metabolism & 13 & 11 & 14 & 11 & - & - & - & - \\
\hline 00770 & Pantothenate and CoA biosynthesis & - & - & - & - & - & - & 30 & 16 \\
\hline 00860 & Porphyrin and chlorophyll metabolism & & & 41 & 33 & 36 & 33 & 50 & 33 \\
\hline 1.9 & Metabolism of terpenoids and polyketides & & & & & & & & \\
\hline 00900 & Terpenoid backbone biosynthesis & - & - & - & - & - & - & 58 & 30 \\
\hline 00904 & Diterpenoid biosynthesis & - & - & - & - & 27 & 18 & - & - \\
\hline 1.10 & Biosynthesis of other secondary metabolites & & & & & & & & \\
\hline 00940 & Phenylpropanoid biosynthesis & - & - & - & - & 114 & 17 & - & - \\
\hline 2. & Genetic Information Processing & & & & & & & & \\
\hline 2.1 & Transcription & & & & & & & & \\
\hline 03040 & Spliceosome & - & - & - & - & - & - & 194 & 102 \\
\hline
\end{tabular}


Table 1 Genes enriched for KEGG and number of KO hits for Juncus effusus, Sorghum bicolor, Oryza sativa, and Zea mays (Continued)

\begin{tabular}{|c|c|c|c|c|c|c|c|c|c|}
\hline \multirow[b]{2}{*}{ Ranking } & \multirow[b]{2}{*}{ KEGG pathway } & \multicolumn{2}{|l|}{ J. effusus } & \multicolumn{2}{|l|}{ S. bicolor } & \multicolumn{2}{|l|}{ O. sativa } & \multicolumn{2}{|l|}{ Z. mays } \\
\hline & & KEGG hits & KO hits & KEGG hits & $\mathrm{KO}$ hits & KEGG hits & $\mathrm{KO}$ hits & KEGG hits & KO hits \\
\hline 2.2 & Translation & & & & & & & & \\
\hline 03010 & Ribosome & 276 & 130 & 278 & 132 & - & - & - & - \\
\hline 03015 & mRNA surveillance pathway & - & - & - & - & 97 & 49 & & \\
\hline 2.3 & Folding, sorting and degradation & & & & & & & & \\
\hline 03060 & Protein export & 46 & 26 & 47 & 26 & - & - & - & - \\
\hline 04122 & Sulfur relay system & - & - & - & - & - & - & 14 & 10 \\
\hline 3. & Environmental Information Processing & & & & & & & & \\
\hline 3.2 & Signal transduction & & & & & & & & \\
\hline 04075 & Plant hormone signal transduction & - & - & - & - & 172 & 38 & 253 & 41 \\
\hline 4. & Cellular Processes & & & & & & & & \\
\hline 4.1 & Transport and catabolism & & & & & & & & \\
\hline 04144 & Endocytosis & - & - & - & - & 117 & 58 & - & - \\
\hline
\end{tabular}

restricted to the plastome [28] and several chromosomal microsatellite loci, the latter of which formed the basis for recent studies on the intraspecific variability of genotypes [46-48]. In order to develop a more comprehensive inventory of genetic information of $J$. effusus we opted for transcriptome profiling of 18 genotypes via RNA-Seq. The geographical origins of the genotypes cover a substantial portion of the European distribution range of this species. RNA-Seq has been used for other members of the Poales, and particular of members of the family Poaceae, for various purposes such as de novo sequencing and assembly (rice, [49]), querying the transcriptome profiles of distinct tissues and at various development stages (wheat; [50]), characterization of genes involved in specific biochemical pathways (cordgrass, [51]; pineapple, [52]), identification of novel transcriptome sequences (maize, [53]) and isoforms (false-brome, [54]), SNP analysis (wheat, [55]), and simple sequence repeats detection (bamboo, [56]).

In this study we carried out both single and pairedend sequencing runs to improve the de novo assembly [57]. The assembly was deemed successful based on a good TransRate score of 0.34 as well BUSCO results that were quite similar to those of the closer relatives $S$. bicolor, O. sativa, and Z. mays. Annotation was performed using the dammit! pipeline (prepared by Camille Scott https://github.com/dib-lab/dammit), which is one of two pipelines available for transcriptome annotation known to us. The other pipeline, annocript [58], was in its earlier stage of development at the time of annotating the J. effusus transcriptome and it did not include information on lower hierarchy of GO terms. The dammit! pipeline includes quality assurance step via BUSCO, executes gene model building [59], and compares each transcript against entries in several databases [e.g. protein domains [60], non-coding RNAs [40], ortholog matches and orthology assignments [61]].

In total, $70 \%$ of the contigs $(111,567)$ were annotated for functional descriptions, GO terms, and conserved protein domains. Sequence similarity searches and gene model building revealed the presence of 120,343 likely coding regions, which were computationally condensed to non-redundant 30,932 gene sequences with matching hits to proteins $\left(E<1 \mathrm{e}^{-6}\right)$. The GO annotation of $J$. effusus transcriptome sequences revealed a similar functional distribution with the reference transcriptomes of $S$. bicolor and $O$. sativa, which evinces overlapping gene complements between $J$. effusus and its relatives. As expected from transcriptome analyses of other members of the Poacaea the most highly represented GO terms belonged to the Molecular Functions category. KEGG pathway analyses mirrored these similarities, and COG analysis matched the topology of the phylogenetic tree based on plastome sequences. Overall, gene function analyses showed that predicted protein sequences exhibited high coverage of KEGG pathways (i.e. 380 KEGG pathways were identified with 7036 enzyme codes). The sequences that had no significant matches may be lacking a known conserved functional domain or they were too short to have a significant sequence match. These sequences might be of potential interests for future research on novel gene products, alternative splice variants, and differentially expressed genes.

A further aim of this study was to identify nucleotide polymorphism markers that can be readily used for genotyping of $J$. effusus. SNPs frequency in the transcriptome ( 1 in $147 \mathrm{bp}$ ) was similar to those of other members of the Poales. SNPs may not be 
Table 2 Candidate genes putatively linked to $\mathrm{N}$ assimilation and associated SNP loci

\begin{tabular}{|c|c|c|c|c|c|}
\hline Contig-ID & Gene family & GO term & Function & Position & SNP \\
\hline Transcript_102832 & alanine aminotransferase & GO:0004021 & aminotransferase & $392 *$ & G/A \\
\hline \multirow[t]{2}{*}{ Transcript_149390 } & \multirow{2}{*}{$\begin{array}{l}\text { ammonium transporter } 1 \\
\text { member }\end{array}$} & \multirow{2}{*}{ GO:0015696 } & \multirow{2}{*}{ ammonium transport } & $618^{*}$ & $\mathrm{G} / \mathrm{A}$ \\
\hline & & & & 328 & $\mathrm{G} / \mathrm{C}$ \\
\hline \multirow{2}{*}{ Transcript_63064 } & \multirow{2}{*}{ ammonium transporter } & \multirow{2}{*}{ GO:0072488 } & \multirow{2}{*}{ ammonium transmembrane transport } & $1655^{*}$ & $\mathrm{C} / \mathrm{T}$ \\
\hline & & & & $306^{*}$ & G/A \\
\hline \multirow[b]{2}{*}{ Transcript_109837 } & \multirow[b]{2}{*}{ aspartate aminotransferase } & \multirow{2}{*}{$\begin{array}{l}\text { GO:0006522, } \\
\text { GO:0006531, } \\
\text { GO:0006536, } \\
\text { GO:0010150 }\end{array}$} & \multirow[b]{2}{*}{$\begin{array}{l}\text { alanine, aspartic and glutamate } \\
\text { metabolic process, leaf senescence }\end{array}$} & $302 *$ & $\mathrm{C} / \mathrm{T}$ \\
\hline & & & & 669 & $\mathrm{G} / \mathrm{A}$ \\
\hline \multirow{3}{*}{ Transcript_76821 } & \multirow{3}{*}{$\begin{array}{c}\text { aspartate kinase-homoserine } \\
\text { dehydrogenase }\end{array}$} & \multirow{3}{*}{ GO:0004072 } & \multirow{3}{*}{ aspartate kinase activity } & $1640^{*}$ & $\mathrm{~A} / \mathrm{G}$ \\
\hline & & & & $1405^{*}$ & $\mathrm{~A} / \mathrm{C}$ \\
\hline & & & & $1842 *$ & $\mathrm{~T} / \mathrm{C}$ \\
\hline \multirow{4}{*}{ Transcript_106397 } & \multirow{4}{*}{ asparagine synthetase } & \multirow{4}{*}{ GO:0097164 } & \multirow{4}{*}{ ammonium ion metabolic process } & $1595^{*}$ & $\mathrm{~T} / \mathrm{A}$ \\
\hline & & & & $2501^{*}$ & $\mathrm{C} / \mathrm{T}$ \\
\hline & & & & 1367 & $\mathrm{C} / \mathrm{A}$ \\
\hline & & & & $1536^{*}$ & $\mathrm{~T} / \mathrm{C}$ \\
\hline \multirow{2}{*}{ Transcript_154402 } & \multirow{2}{*}{ Ferredoxin NAPD + reductase } & \multirow{2}{*}{ GO:0004324; } & \multirow{2}{*}{$\begin{array}{l}\text { ferredoxin-NADP+ reductase } \\
\text { activity }\end{array}$} & $1068^{*}$ & $\mathrm{~T} / \mathrm{A}$ \\
\hline & & & & $283 *$ & $\mathrm{~T} / \mathrm{C}$ \\
\hline & & & & $775^{*}$ & $\mathrm{~A} / \mathrm{G}$ \\
\hline Transcript_56560 & Ferredoxin-nitrite reductase & GO:0048307 & ferredoxin-nitrite reductase activity & $1317 *$ & $\mathrm{C} / \mathrm{A}$ \\
\hline & & & & $1014^{*}$ & $\mathrm{G} / \mathrm{T}$ \\
\hline & & GO:0004356, & glutamate-ammonia ligase activity, & $1358^{*}$ & $\mathrm{~T} / \mathrm{C}$ \\
\hline Transcript_132440 & glutamine synthetase & GO:0009399 & nitrogen fixation & $414^{*}$ & $\mathrm{C} / \mathrm{T}$ \\
\hline & & & & 616 & $\mathrm{G} / \mathrm{A}$ \\
\hline Transcript 140124 & glutamine synthetase & GO:0004356 & glutamate-ammonia ligase activity & $1170^{*}$ & $\mathrm{G} / \mathrm{T}$ \\
\hline & & & & $1905^{*}$ & $\mathrm{~A} / \mathrm{T}$ \\
\hline Transcript_81145 & glutamate synthase 1 & GO:0060359 & response to ammonium ion & $1417^{*}$ & $\mathrm{~T} / \mathrm{C}$ \\
\hline & & & & $2624 *$ & $\mathrm{C} / \mathrm{T}$ \\
\hline Transcrint 132300 & olutamine sunthetase & G0.0042128 & & $204 *$ & $\mathrm{~A} / \mathrm{T}$ \\
\hline Transcript_132390 & glutamine synthetase & $\mathrm{GO}: 0042128$ & nitrate assimilation & $472 *$ & $\mathrm{~A} / \mathrm{G}$ \\
\hline & & & & 468 & $\mathrm{G} / \mathrm{C}$ \\
\hline & & & & 393 & $\mathrm{C} / \mathrm{T}$ \\
\hline Transcript_82003 & glutamine synthetase & GO:0004356 & glutamate-ammonia ligase activity & 416 & $\mathrm{G} / \mathrm{A}$ \\
\hline & & & & 352 & $\mathrm{C} / \mathrm{T}$ \\
\hline Transcrint 132200 & glutamate dehydrogenase & GO:0006995, & Response to nitrogen compound & $973 *$ & $\mathrm{~A} / \mathrm{G}$ \\
\hline Iranscript_132200 & glutamate dehydrogenase & GO: 1901698 & Response to nitrogen compound & 494* & $\mathrm{A} / \mathrm{G}$ \\
\hline & & & & $254^{*}$ & $\mathrm{~T} / \mathrm{A}$ \\
\hline Transcript_53371 & nitrate reductase & GO:0042128 & nitrate assimilation & 149 & $\mathrm{~T} / \mathrm{C}$ \\
\hline & & & & $628^{*}$ & $\mathrm{~A} / \mathrm{G}$ \\
\hline Transcrint 156517 & nitrate transnorter -like & GO·.0010167 & resnonse to nitrate & $314^{*}$ & $\mathrm{G} / \mathrm{A}$ \\
\hline & & & & $162 *$ & $\mathrm{G} / \mathrm{A}$ \\
\hline & & & & $1199 *$ & $\mathrm{C} / \mathrm{T}$ \\
\hline Transcript_136631 & NRT1/peptide transporter & GO:0006807 & nitrogen compound metabolic & $563 *$ & $\mathrm{~A} / \mathrm{C}$ \\
\hline & & & & $390^{*}$ & $\mathrm{C} / \mathrm{T}$ \\
\hline Transcrint 138058 & & $G 0 \cdot 0015706$ & & $464^{*}$ & $\mathrm{G} / \mathrm{A}$ \\
\hline Iranscript_138058 & vacuolar-sortıng receptor & GO:0015/06 & nitrate transport & $442^{*}$ & $\mathrm{~T} / \mathrm{C}$ \\
\hline
\end{tabular}

SNPs marked in orange are located at synonymous codon-positions. An asterisk marks successful PCR amplification

distributed evenly across the genome positionally and functionally. For example, in soy bean SNPs were found to occur more frequently at the chromosome ends putatively as a consequence of diversifying recombination and mutation events [62]. Without reference genome for J. effusus, however, the chromosomal distribution of the found SNPs cannot be assessed properly. At the functional level, we found the highest SNP frequency per locus in transcripts involved in plant defense mechanisms and wound healing. In O. sativa, a large amount of SNP variation was found in genes involved in in stress response and other processes associated with adaptation to a changing environment [63]. Similarly, for A. thaliana it has been suggested that the maintenance of variation in defense mechanisms may be associated with the species' persistence in environmentally heterogeneous habitats [64]. Hence, also $J$. effusus as a wetland indicator plant and tolerating a wide range of ecological conditions may benefit from a diverse genomic background associated with stress tolerance. The polymorphisms identified here at transcriptome level will help explain adaptive mechanisms shaping and maintaining the complex intra-specific differentiation pattern described for this species [24]. 


\section{Conclusions}

This study is the first genomics analysis of J. effusus. The assembly and annotation were considered as of high quality. The results are expected to open new opportunities for future omics studies and population genetics of this common wetland plant.

\section{Materials and methods}

\section{Plant materials and RNA isolation}

Plant tissues (roots and shoots) were harvested from individuals at the vegetative developmental stage that were raised from seeds collected in the field. In total 18 genotypes were used. The geographical distribution of the sampled locations is presented in Fig. 4. The obtained plant tissues were snap-frozen in liquid nitrogen and kept at $-80^{\circ} \mathrm{C}$ until processing. Total RNA was extracted from roots and shoots separately using the RNeasy Plant Mini Kit according to the manufacturer's protocol (Qiagen, Hilden, Germany). In order to represent a wide range of expressed genotypic variability within individuals and the species, extracts were then pooled with the final mix containing approximate equal contributions of each genotype and tissue type.

\section{Transcriptome sequencing and assembly}

Standard library preparation and sequencing of total RNA using one lane of an Illumina HiSeq $(2 \times 100 \mathrm{bp}$ $\mathrm{PE})$ and two runs of Roche 454 Titanium was done at the Duke Center for Genomic and Computational Biology (Durham, USA) yielding 249 million Illumina $\mathrm{PE}$ reads and 2.8 million 454 reads. After removing sequencing adaptors, quality-controlled reads were processed using two different de novo transcriptome assemblers. Illumina reads were assembled using Trinity version 20,130,225 [29], and 454 reads were assembled using Mira version 3.9.15, [30, 31]. Both assemblers were run with default parameters. The software TransRate [65], which enables reference-free quality evaluations of de novo transcriptome assemblies, was used for analysis of Trinity assembly. Mira and Trinity assemblies were combined and CD-HIT version 4.5.7 [32, 33] was used to remove redundant sequences.

\section{Functional annotation}

We used Camille Scott's dammit! annotation pipeline to annotate the transcriptome assembly (https://github. com/camillescott/dammit). Within the pipeline, annotation begins by building gene models with TransDecoder v2.0.1 [35]. Subsequently, it utilizes multiple databases for annotating the transcriptome: protein domains in Pfam-A v29.0 [36, 37], Rfam v12.0 to find non-coding RNAs [40], the execution of a LAST search for known proteins in the OrthoDB database [61], ortholog matches in the BUSCO database [34], and orthology searches in OrthoDB [61]. The assembly quality and annotation completeness were assessed using BUSCO v3, which supports interpretation of assembly coverage based on the presence of single-copy orthologous genes [34]. To compare the assembly results of $J$. effusus, BUSCO was also run with transcriptomes of O. sativa subsp. japonica and S. bicolor (http://plants.ensembl.org/info/website/ ftp/index.html).

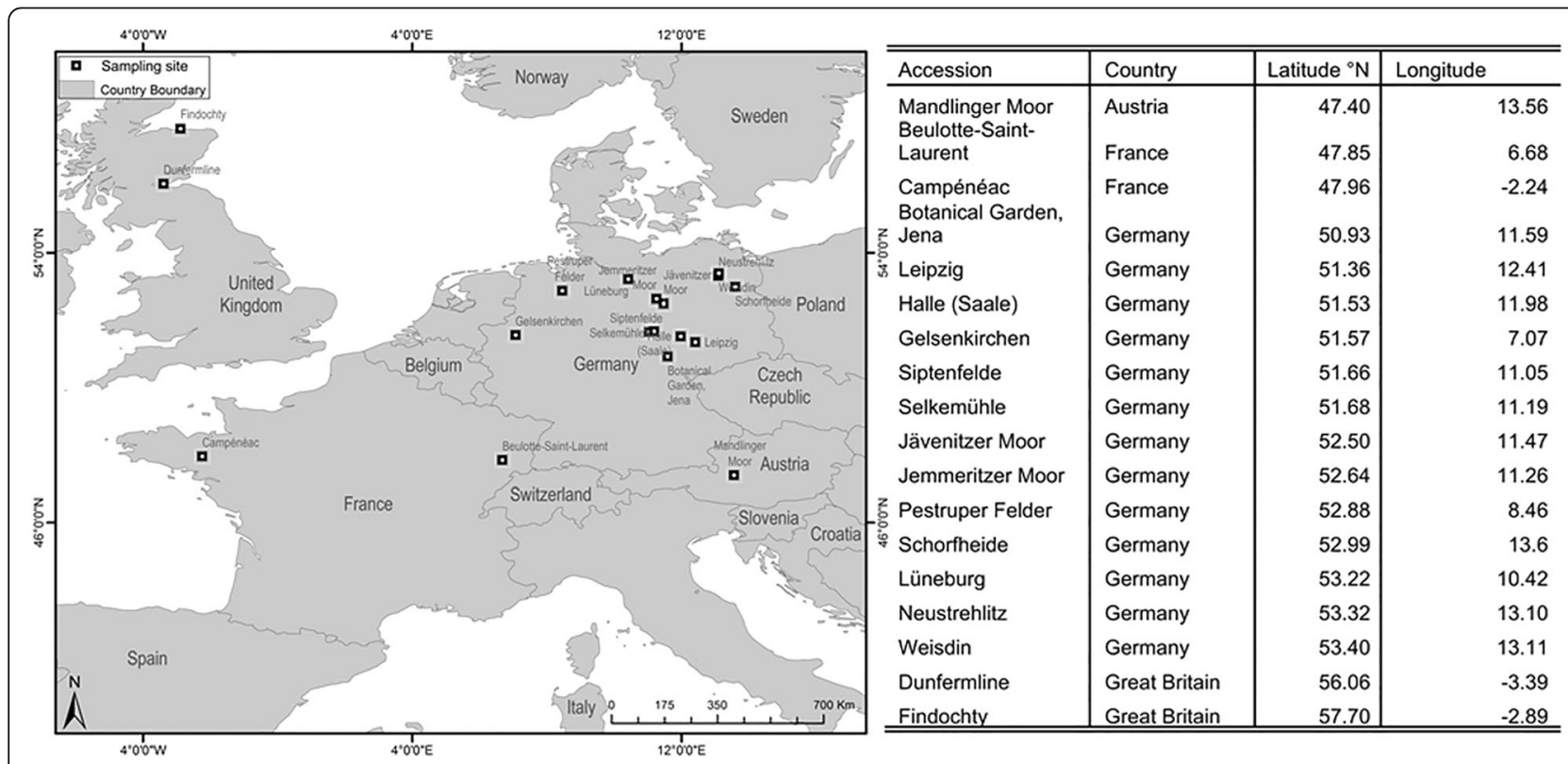

Fig. 4 Overview map indicating sampling sites for $J$. effusus ecotypes analysed in this study 


\section{Functional classification}

Gene ontology (GO) analyses were carried out on predicted protein sequences using InterProScan v.5.26-65.0, available as virtual machine image on Jetstream cloud (https://use. jetstream-cloud.org/application/images/586). The GO annotations were then plotted using the BGI-WEGO program (http://wego.genomics.org.cn/) together with O. sativa and $S$. bicolor to elucidate relative distribution of Molecular Function, Cellular Components, and Biological Processes [41]. Afterwards, predicted protein sequences were mapped to the reference canonical Kyoto Encyclopedia Genes and Genomes (KEGG) pathways as additional approach for functional annotation and categorization. The predicted protein sequences were submitted to the KEGG automatic annotation server (KAAS) (http://www.genome.jp/tools/ kaas/) with the single-directional best-hit (SBH) method selected for pathway mapping. Subsequently, gene set enrichment analysis (GSEA) was performed on non-redundant gene sequences using the GO based enrichment tool DAVID (Database for Annotation, Visualization and Integrated Discovery) [43]. DAVID provides ranking of KEGG pathways on the basis of Benjamini corrected $p$-values. The number of genes shared between $J$. effusus and the members of the Poaceae S. bicolor, O. sativa, and Z. mays were assessed by OrthoVenn, a web platform that identifies COGs clusters by comparing the predicted proteins sequences with the database [66]. Default parameters were used for protein similarity comparisons.

\section{Polymorphism identification}

Paired end Illumina reads were mapped using segemehl version 0.1.9 (Hoffmann et al., 2009) against the de novo transcriptome assembly allowing two mismatches as well as multiple mappings to redundant sequences in the variable mapping seed. Subsequently single nucleotide polymorphisms (SNPs) and insertions/deletions (INDELS) were detected using samtools mpileup (version 1.1) [67] using default parameters. SNPs were annotated using Ensemble Variant Effect Predictor (version 75) [68]. Contigs with significant SNPs (3-fold greater abundance than the transcriptome-wide average) were identified and then $\mathrm{KO}$ assigned using concatenate and merge function in R-statistical language. Lastly, KO terms were annotated by using the online KEGG database (https://www.genome.jp/kegg/ko.html). Selected SNP loci putatively associated with $\mathrm{N}$ assimilation were verified by the following approach: For the same 18 genotypes used for total RNA extraction, genomic DNA was extracted using the DNeasy Plant Mini Kit according to the manufacturer's protocol (Qiagen, Hilden, Germany). Primers for each locus were designed using a Primer3 online implementation (http:// bioinfo.ut.ee/primer3-0.4.0/) targeting a size range between 300 and $600 \mathrm{bp}$. Amplification of loci was conducted in a total volume of $20 \mu \mathrm{L}$ including 5 pMol of each locus-specific forward and reverse primer, $200 \mu \mathrm{M}$ dNTP, $2 \mu \mathrm{L} \quad 10 \mathrm{x} \quad$ DreamTaq-buffer (Thermo Fischer Scientific), $0.8 \mathrm{U}$ DreamTaqPolymerase and approx. $5 \mathrm{ng}$ DNA. The PCR program involved $3 \mathrm{~min}$ at $95^{\circ} \mathrm{C}$, then 40 cycles of $95^{\circ} \mathrm{C}$ for $30 \mathrm{~s}, 58-62^{\circ} \mathrm{C}$ for $40 \mathrm{~s}, 72^{\circ} \mathrm{C}$ for $1 \mathrm{~min}$ and a final 10 min of $72^{\circ} \mathrm{C}$. PCRs products were purified by centrifuging for $3 \mathrm{~min}$ at $2800 \mathrm{rpm}$ through cross linked dextran gel (Sephadex G-50 Superfine, GE Healthcare Life Sciences, Germany). PCR-products were directly cycle-sequenced from both ends using the $\mathrm{ABI}$ BigDye Terminater v3.1 cycle sequencing Kit using the same primers. Products were sequenced on an Applied Biosystems 3130xl Genetic Analyzer (Applied Biosystems, Foster City, USA).

\section{Abbreviations}

BUSCO: Benchmarking Single-Copy Ortholog genes; EIP: environmental information processing; GSEA: Gene Set Enrichment Analysis; INDELs: insertions/deletions; SNPS: single nucleotide polymorphisms; UTR: untranslated region

\section{Acknowledgments}

The authors thank Dr. Saddam Akber Abbasi (Assistant Professor, Department of Mathematics and Statistics, Qatar University, Doha, Qatar) for his help in statistical analysis and writing scripts.

\section{Research involving plants}

Juncus effusus is not endangered or a protected species in Europe, and no permissions for collecting seeds in the wild were required. Specimens were collected and identified taxonomically by SGM, who is a botanist with advanced expertise on this species.

\section{Authors' contributions}

SGM, MA, IG and JAM planned the study. MA, MP, and UKD with assistance by IG, SGM, and JAM analysed the data. MA and JAM wrote the first draft of the paper and all authors assisted with the development of the final draft. All authors read and approved the final manuscript.

\section{Funding}

MA was supported by the German Academic Exchange Program (DAAD). SGM acknowledges funding by the German Science Foundation (DFG, Grant No. MI 1500/2-1).

\section{Availability of data and materials}

The assembly is available at GenBank under accession no. PRJNA345287. Al annotation files incl. Supplementary Tables are available at Mendely under the DOl:https://doi.org/10.17632/cx7k2v38m7.3.

Ethics approval and consent to participate

Not applicable.

Consent for publication

Not applicable.

\section{Competing interests}

The authors declare that they have no competing interests.

\section{Author details}

'Department Environmental Biotechnology, Helmholtz Centre for Environmental Research - UFZ, Permoserstr, 15 Leipzig, Germany. ${ }^{2}$ Institute for Biology $V$ (Environmental Research), RWTH Aachen University, Templergraben 55, 52062 Aachen, Germany. ${ }^{3} \mathrm{BIO} 5$ Institute, The University of Arizona, Tucson, AZ 85719, USA. ${ }^{4}$ Institute of Computer Science, 
Martin-Luther-University Halle-Wittenberg, Von-Seckendorff-Platz 1, 06120 Halle (Saale), Germany. ${ }^{5}$ Core Facility Deep Sequencing,

Martin-Luther-University Halle-Wittenberg, Magdeburger Str. 2, 06112 Halle (Saale), Germany. ${ }^{6}$ German Centre for Integrative Biodiversity Research (iDiv) Halle-Jena-Leipzig, Deutscher Platz 5e, 04103 Leipzig, Germany. ${ }^{7}$ Department of Community Ecology, Helmholtz Centre for Environmental Research - UFZ, Theodor-Lieser-Str. 4, 06120 Halle (Saale), Germany.

Received: 23 July 2018 Accepted: 5 June 2019

Published online: 13 June 2019

\section{References}

1. Kirschner J. Juncaceae, vol. 3: Australian Biological Resources Study; 2002.

2. Michalski SG, Durka W. Separation in flowering time contributes to the maintenance of sympatric cryptic plant lineages. Ecol Evol. 2015;5:2172-84.

3. Richards P, Clapham A. Juncus effusus L.(Juncus communis $\beta$ effusus E. Mey). J Ecol. 1941;29:375-80.

4. Visser EJ, Bögemann GM. Aerenchyma formation in the wetland plant Juncus effusus is independent of ethylene. New Phytol. 2006;171:305-14.

5. Vymazal J. Constructed wetlands for treatment of industrial wastewaters: a review. Ecol Eng. 2014;73:724-51.

6. Vymazal J, Březinová T. Accumulation of heavy metals in aboveground biomass of Phragmites australis in horizontal flow constructed wetlands for wastewater treatment: a review. Chem Eng J. 2016;290:232-42.

7. Wiessner A, Kuschk P, Jechorek M, Seidel H, Kästner M. Sulphur transformation and deposition in the rhizosphere of Juncus effusus in a laboratory-scale constructed wetland. Enviro Pollut. 2008;155:125-31.

8. Bhullar GS, Edwards PJ, Venterink HO. Influence of different plant species on methane emissions from soil in a restored Swiss wetland. PLoS One. 2014;9: e89588.

9. Colmer T. Long-distance transport of gases in plants: a perspective on internal aeration and radial oxygen loss from roots. Plant Cell Environ. 2003;26:17-36.

10. Syranidou E, Christofilopoulos S, Kalogerakis N. Juncus spp.- -the helophyte for all (phyto) remediation purposes? New Biotechnol. 2017;38:43-55.

11. Henneberg A, Brix H, Sorrell BK. The interactive effect of Juncus effusus and water table position on mesocosm methanogenesis and methane emissions. Plant Soil. 2016:400:45-54.

12. Saarnio $S$, Wittenmayer $L$, Merbach $W$. Rhizospheric exudation of Eriophorum vaginatum L.- - potential link to methanogenesis. Plant Soil. 2004;267:343-55.

13. Henneberg A, Sorrell BK, Brix H. Internal methane transport through Juncus effusus: experimental manipulation of morphological barriers to test above-and below-ground diffusion limitation. New Phytol. 2012;196: 799-806.

14. Stottmeister U, Wießner A, Kuschk P, Kappelmeyer U, Kästner M, Bederski O, Müller $\mathrm{R}$, et al. Effects of plants and microorganisms in constructed wetlands for wastewater treatment. Biotechnol Adv. 2003;22:93-117.

15. Agethen S, Knorr K-H. Juncus effusus mono-stands in restored cutover peat bogs - analysis of litter quality, controls of anaerobic decomposition, and the risk of secondary carbon loss. Soil Biol Biochem. 2018;117:139-52.

16. Martínez-Lavanchy P, Chen Z, Lünsmann V, Marin-Cevada V, Vilchez-Vargas R, Pieper D, Reiche N, Kappelmeyer U, et al. Microbial toluene removal in hypoxic model constructed wetlands occurs predominantly via the ring monooxygenation pathway. Appl Environ Microbiol. 2015;81:6241-52.

17. Rahman KZ, Wiessner A, Kuschk P, van Afferden M, Mattusch J, Müller RA. Removal and fate of arsenic in the rhizosphere of Juncus effusus treating artificial wastewater in laboratory-scale constructed wetlands. Ecol Eng. 2014;69:93-105.

18. Wiessner A, Kuschk P, Nguyen PM, Müller JA. The sulfur depot in the rhizosphere of a common wetland plant, Juncus effusus, can support long-term dynamics of inorganic sulfur transformations. Chemosphere. 2017;184:375-83.

19. Xu L, Najeeb U, Raziuddin R, Shen W, Shou J, Tang G, Zhou W. Development of an efficient tissue culture protocol for callus formation and plant regeneration of wetland species Juncus effusus L. In Vitro Cell Dev Biol Plant. 2009:45:610-8.

20. Ishiuchi Ki KY, Hamagami H, Ozaki M, Ishige K, Ito Y, Kitanaka S. Chemical constituents isolated from Juncus effusus induce cytotoxicity in HT22 cells. J Nat Med. 2015;69:421-6.
21. Liao Y-J, Zhai H-F, Zhang B, Duan T-X, Huang J-M. Anxiolytic and sedative effects of dehydroeffusol from Juncus effusus in mice. Planta Med. 2011;77:416-20.

22. Singhuber J, Baburin I, Khom S, Zehl M, Urban E, Hering S, Kopp B. GABA receptor modulators from the Chinese herbal drug Junci medulla-the pith of Juncus effusus. Planta Med. 2012;78:455-8.

23. Berger J, Suzuki T, Senti K-A, Stubbs J, Schaffner G, Dickson BJ. Genetic mapping with SNP markers in Drosophila. Nature Genet. 2001;29:475.

24. Michalski SG, Durka W. Identification and characterization of microsatellite loci in the rush Juncus effusus (Juncaceae). Am J Bot. 2012;99:e53-5.

25. Bennett M, Leitch I. Plant DNA C-values database. In: Royal Botanic Gardens Kew. 2005.

26. Goff SA, Ricke D, Lan T-H, Presting G, Wang R, Dunn M, Glazebrook J, et al. A draft sequence of the rice genome (Oryza sativa L. ssp. japonica). Science. 2002;296:92-100.

27. Yu J, Hu S, Wang J, Wong GK-S, Li S, Liu B, Deng Y, et al. A draft sequence of the rice genome (Oryza sativa L. ssp. indica). Science. 2002;296:79-92.

28. Givnish TJ, Ames M, McNeal JR, McKain MR, Steele PR, Depamphilis CW, Graham SW, et al. Assembling the tree of the monocotyledons: plastome sequence phylogeny and evolution of Poales. Ann Missouri Bot Gard. 2010; 97:584-616.

29. Grabherr MG, Haas BJ, Yassour M, Levin JZ, Thompson DA, Amit I, Adiconis $X$, et al. Trinity: reconstructing a full-length transcriptome without a genome from RNA-Seq data. Nature Biotechnol. 2011;29:644-52.

30. Chevreux B, Pfisterer T, Drescher B, Driesel AJ, Müller WE, Wetter T, Suhai S. Using the miraEST assembler for reliable and automated mRNA transcript assembly and SNP detection in sequenced ESTs. Genome Res. 2004;14: 1147-59.

31. Chevreux B, Wetter T, Suhai S. Genome sequence assembly using trace signals and additional sequence information. In: German conference on bioinformatics: 1999: Citeseer; 1999: 45-56.

32. Fu L, Niu B, Zhu Z, Wu S, Li W. CD-HIT: accelerated for clustering the nextgeneration sequencing data. Bioinformatics. 2012;28:3150-2.

33. Li W, Godzik A. Cd-hit: a fast program for clustering and comparing large sets of protein or nucleotide sequences. Bioinformatics. 2006;22:1658-9.

34. Simão FA, Waterhouse RM, loannidis P, Kriventseva EV, Zdobnov EM. BUSCO: assessing genome assembly and annotation completeness with single-copy orthologs. Bioinformatics. 2015;31:3210-2.

35. Haas BJ, Papanicolaou A, Yassour M, Grabherr M, Blood PD, Bowden J, Couger MB, et al. De novo transcript sequence reconstruction from RNA-seq using the trinity platform for reference generation and analysis. Nat Protoc. 2013;8:1494-512.

36. Finn RD, Coggill P, Eberhardt RY, Eddy SR, Mistry J, Mitchell AL, Potter SC, et al. The Pfam protein families database: towards a more sustainable future. Nucleic Acids Res. 2015:44:D279-85.

37. Sonnhammer EL, Eddy SR, Durbin R. Pfam: a comprehensive database of protein domain families based on seed alignments. Proteins. 1997;28: 405-20.

38. Suzek BE, Huang H, McGarvey P, Mazumder R, Wu CH. UniRef: comprehensive and non-redundant UniProt reference clusters. Bioinformatics. 2007;23:1282-8.

39. Suzek BE, Wang Y, Huang H, McGarvey PB, Wu CH, Consortium U. UniRef clusters: a comprehensive and scalable alternative for improving sequence similarity searches. Bioinformatics. 2014;31:926-32.

40. Nawrocki EP, Burge SW, Bateman A, Daub J, Eberhardt RY, Eddy SR, Floden EW, et al. Rfam 12.0: updates to the RNA families database. Nucleic Acids Res. 2014;43:D130-7.

41. Ye J, Fang L, Zheng H, Zhang Y, Chen J, Zhang Z, Wang J, et al. WEGO: a web tool for plotting GO annotations. Nucleic Acids Res. 2006;34:W293-7.

42. Ren C-G, Kong C-C, Yan K, Zhang H, Luo Y-M, Xie Z-H. Elucidation of the molecular responses to waterlogging in Sesbania cannabina roots by transcriptome profiling. Sci Rep. 2017;7:9256.

43. Huang DW, Sherman BT, Zheng X, Yang J, Imamichi T, Stephens R, Lempicki RA. Extracting biological meaning from large gene lists with DAVID. Curr Protoc Bioinformatics. 2009;27:13-1.

44. Subbaiyan GK, Waters DL, Katiyar SK, Sadananda AR, Vaddadi S, Henry RJ. Genome-wide DNA polymorphisms in elite indica rice inbreds discovered by whole-genome sequencing. Plant Biotechnol J. 2012;10:623-34.

45. Gore MA, Wright MH, Ersoz ES, Bouffard P, Szekeres ES, Jarvie TP, Hurwitz $B L$, et al. Large-scale discovery of gene-enriched SNPS. Plant Genome. 2009;2:121-33. 
46. Born J, Michalski SG. Strong divergence in quantitative traits and plastic behavior in response to nitrogen availability among provenances of a common wetland plant. Aquat Bot. 2017;136:138-45.

47. Born J, Michalski SG. Trait expression and signatures of adaptation in response to nitrogen addition in the common wetland plant Juncus effusus. PLoS One. 2019;14:e0209886.

48. Michalski SG, Durka W. Identification and characterization of microsatellite loci in the rush Juncus effusus (Juncaceae) 1. Am J Bot. 2012;99:e53-5.

49. X-j T, Long Y, Wang J, J-w Z, Y-y W, Li W-m, Y-f P, et al. De novo transcriptome assembly of common wild rice (Oryza rufipogon Griff.) and discovery of drought-response genes in root tissue based on transcriptomic data. PLoS One. 2015;10:e0131455.

50. Chu Z, Chen J, Sun J, Dong Z, Yang X, Wang Y, Xu H, et al. De novo assembly and comparative analysis of the transcriptome of embryogenic callus formation in bread wheat (Triticum aestivum L.). BMC Plant Biol. 2017;17:244.

51. De Carvalho JF, Poulain J, Da Silva C, Wincker P, Michon-Coudouel S, Dheilly A, Naquin D, et al. Transcriptome de novo assembly from nextgeneration sequencing and comparative analyses in the hexaploid salt marsh species Spartina maritima and Spartina alterniflora (Poaceae). Heredity. 2013;110:181.

52. Ma J, Kanakala S, He Y, Zhang J, Zhong X. Transcriptome sequence analysis of an ornamental plant, Ananas comosus var. bracteatus, revealed the potential unigenes involved in terpenoid and phenylpropanoid biosynthesis. PLoS One. 2015;10:e0119153.

53. Hansey CN, Vaillancourt B, Sekhon RS, De Leon N, Kaeppler SM, Buell CR. Maize (Zea mays L.) genome diversity as revealed by RNA-sequencing. PLoS One. 2012;7:e33071.

54. Fox SE, Preece J, Kimbrel JA, Marchini GL, Sage A, Youens-Clark K, Cruzan $\mathrm{MB}$, et al. Sequencing and de novo transcriptome assembly of Brachypodium sylvaticum (Poaceae). Appl Plant Sci. 2013;1:1200011.

55. Fox SE, Geniza M, Hanumappa M, Naithani S, Sullivan C, Preece J, Tiwari VK, et al. De novo transcriptome assembly and analyses of gene expression during photomorphogenesis in diploid wheat Triticum monococcum. PLoS One. 2014;9:e96855.

56. Liu M, Qiao G, Jiang J, Yang H, Xie L, Xie J, Zhuo R. Transcriptome sequencing and de novo analysis for ma bamboo (Dendrocalamus latiflorus Munro) using the Illumina platform. PLoS One. 2012;7:e46766.

57. Cahais V, Gayral P, Tsagkogeorga G, Melo-Ferreira J, Ballenghien M, Weinert $L$, Chiari $Y$, et al. Reference-free transcriptome assembly in non-model animals from next-generation sequencing data. Mol Ecol Resour. 2012;12: 834-45.

58. Musacchia F, Basu S, Petrosino G, Salvemini M, Sanges R. Annocript: a flexible pipeline for the annotation of transcriptomes able to identify putative long noncoding RNAs. Bioinformatics. 2015;31:2199-201.

59. Stein LD, Mungall C, Shu S, Caudy M, Mangone M, Day A, Nickerson E, et al. The generic genome browser: a building block for a model organism system database. Genome Res. 2002;12:1599-610.

60. Haas J, Roth S, Arnold K, Kiefer F, Schmidt T, Bordoli L, Schwede T. The protein model portal-a comprehensive resource for protein structure and model information. Database. 2013;2013.

61. Kriventseva EV, Tegenfeldt F, Petty TJ, Waterhouse RM, Simao FA, Pozdnyakov IA, loannidis $P$, et al. OrthoDB v8: update of the hierarchical catalog of orthologs and the underlying free software. Nucleic Acids Res. 2014;43:D250-6.

62. Vidal RO, do Nascimento LC, Mondego JMC, Pereira GAG, Carazzolle MF. Identification of SNPs in RNA-seq data of two cultivars of Glycine max (soybean) differing in drought resistance. Genet Mol Biol. 2012;35:331-4.

63. Tatarinova TV, Chekalin E, Nikolsky Y, Bruskin S, Chebotarov D, McNally KL, Alexandrov N. Nucleotide diversity analysis highlights functionally important genomic regions. Sci Rep. 2016;6:35730.

64. Kerwin R, Feusier J, Corwin J, Rubin M, Lin C, Muok A, Larson B, et al. Natural genetic variation in Arabidopsis thaliana defense metabolism genes modulates field fitness. Elife. 2015;13:e05604

65. Smith-Unna R, Boursnell C, Patro R, Hibberd JM, Kelly S. TransRate: reference-free quality assessment of de novo transcriptome assemblies. Genome Res. 2016;26:1134-44.

66. Wang Y, Coleman-Derr D, Chen G, Gu YQ. OrthoVenn: a web server for genome wide comparison and annotation of orthologous clusters across multiple species. Nucleic Acids Res. 2015;43:W78-84.
67. Li H, Handsaker B, Wysoker A, Fennell T, Ruan J, Homer N, Marth G, et al. The sequence alignment/map format and SAMtools. Bioinformatics. 2009;25:2078-9.

68. McLaren W, Pritchard B, Rios D, Chen Y, Flicek P, Cunningham F. Deriving the consequences of genomic variants with the Ensembl API and SNP effect predictor. Bioinformatics. 2010;26:2069-70.

\section{Publisher's Note}

Springer Nature remains neutral with regard to jurisdictional claims in published maps and institutional affiliations.
Ready to submit your research? Choose BMC and benefit from:

- fast, convenient online submission

- thorough peer review by experienced researchers in your field

- rapid publication on acceptance

- support for research data, including large and complex data types

- gold Open Access which fosters wider collaboration and increased citations

- maximum visibility for your research: over $100 \mathrm{M}$ website views per year

At BMC, research is always in progress.

Learn more biomedcentral.com/submissions 